\title{
M. LOREA
}

\section{La structure de certains problèmes d'affectation}

Revue française d'automatique, d'informatique et de recherche opérationnelle. Recherche opérationnelle, tome 10, nº V3 (1976), p. 59-63.

<http://www.numdam.org/item?id=RO_1976_10_3_59_0>

(C) AFCET, 1976, tous droits réservés.

L'accès aux archives de la revue « Revue française d'automatique, d'informatique et de recherche opérationnelle. Recherche opérationnelle » implique l'accord avec les conditions générales d'utilisation (http://www.numdam.org/ legal.php). Toute utilisation commerciale ou impression systématique est constitutive d'une infraction pénale. Toute copie ou impression de ce fichier doit contenir la présente mention de copyright.

\section{Numdam}

Article numérisé dans le cadre du programme

Numérisation de documents anciens mathématiques

http://www.numdam.org/ 


\title{
LA STRUCTURE DE CERTAINS PROBLEMES D'AFFECTATION $\left({ }^{*}\right)$
}

\author{
par M. LOREA $\left({ }^{1}\right)$
}

Résumé. - Dans cet article, l'importance du concept algébrique de matroide pour l'étude et la résolution de certains problèmes d'affectation est soulignée. Le problème présenté dans [5] est généralisé et on prouve que la théorie des matroides en permet une résolution aisée. Ensuite, on rappelle que la théorie de l'intersection de matroides, bien qu'elle ne mène pas directement à une solution, met en évidence la structure de beaucoup de problèmes d'affectation et les liens qu'ils ont avec d'autres problèmes de recherche opérationnelle.

\section{DÉFINITION D'UN MATROÏDE ([1] et [2])}

Soit $E$ un ensemble fini et $\mathscr{F}$ un ensemble de parties de $E$. Les éléments de $\mathscr{F}$ sont les ensembles indépendants d'un matroïde dans $E$ si et seulement s'ils satisfont aux trois propriétés suivantes :

$$
\begin{aligned}
& 1^{\circ} \varphi \in \mathscr{F} ; \\
& \begin{aligned}
2^{\circ} F \in \mathscr{F} \mid \\
\left.F^{\prime} \subset F\right\}
\end{aligned} \\
& \left.\begin{array}{rl}
3^{\circ} \forall S \subset E: & F \in \mathscr{F} \text {; et } F \text { maximal avec ces deux } \\
& F \subset S \text {; propriétés } \\
& F^{\prime} \in \mathscr{F} ; \text { et } F^{\prime} \text { maximal avec ces } \operatorname{deux} \\
& F^{\prime} \subset S ; \text { propriétés }
\end{array}\right\} \Rightarrow|F|=\left|F^{\prime}\right| .
\end{aligned}
$$

Le couple $M(E, \mathscr{F})$ constitue alors un matroïde. Un ensemble indépendant maximal est appelé une base.

\section{UN THÉORÈME GÉNÉRATEUR DE MATROÏDES [2]}

Soit $E$ un ensemble fini; notons $\mathscr{P}(E)$ l'ensemble des parties $E$ et $N$ l'ensemble des nombres entiers non négatifs.

Soit $f$ une fonction de $\mathscr{P}(E)$ vers $N$ satisfaisant aux trois propriétés suivantes :

$1^{\circ} f(\varphi)=0$;

$2^{\circ} f(A) \geqq f(B), \forall A, B, B \subset A \subset E$;

$3^{\circ} f(A)+f(B) \geqq f(A \cup B)+f(A \cap B), \forall A, B, A \subset E, B \subset E$.

$\left({ }^{*}\right)$ Reçu mai 1975.

(') Chercheur F.R.F.C., Université libre de Bruxelles.

Revue Française d'Automatique, Informatique et Recherche Opérationnelle, octobre 1976. 
THÉORÈME [2] : A partir d'une telle fonction, il est possible de définir un matroïde dans $E$, en prenant pour ensembles indépendants de ce matroïde, les ensembles $X(X \subset E)$ tels que

$$
\forall Y \subset X, \quad f(Y) \geqq|Y| .
$$

\section{UNE IMPORTANTE PROPRIÉTÉ DES MATROÏDES ([1] et [2])}

REMARQUe PRÉliminaIRE : Si $E$ est muni d'un ordre total, il est parfois impossible de comparer deux parties de $E$, de même cardinalité, en utilisant uniquement l'information fournie par l'ordre sur $E$.

Par exemple, si $E=\left\{e_{1}, e_{2}, e_{3}, e_{4}\right\}$ et si l'ordre est $\left[e_{i}>e_{j}(\forall i<j)\right]$, l'information fournie ne permet :

ni d'affirmer que le couple $\left(e_{1}, e_{4}\right)$ est meilleur que $\left(e_{2}, e_{3}\right)$;

ni d'affirmer que le couple $\left(e_{2}, e_{3}\right)$ est meilleur que $\left(e_{1}, e_{4}\right)$.

Le théorème suivant affirme qu'une situation comme celle-ci ne se rencontre pas lors de la recherche de la « meilleure » base d'un matroïde dans un ensemble totalement ordonné $E$.

THÉORÈME : Dans un matroïde $(E, \mathscr{F})$ dans un ensemble E totalement ordonné, il existe une base $B$ telle que, pour toute autre base $B^{*}$, il existe une bijection $g$ : $B \stackrel{g}{\rightarrow} B^{*}$, telle que $b>g(b)$, ou $b=g(b), \forall b \in B$ et telle que $\exists b_{0} \in B$, $b_{0}>g\left(b_{0}\right)$.

On trouve dans [1] la démonstration de ce théorème et un algorithme simple permettant la construction de cette meilleure base $B$.

\section{HYPERGRAPHES ET MATROÏDES ([8] et [9])}

Soit $X$ un ensemble fini et soit $\mathscr{E} \subset \mathscr{P}(X)$.

$H(X, \mathscr{E})$ est appelé un hypergraphe (C. Berge impose des conditions sur l'ensemble $\mathscr{E}$, mais elles ne sont pas indispensables pour ce qui suit).

$A H(X, \mathscr{E})$ et à un couple de nombres entiers $(a, b)(a>0)$, nous associons un matroïde $M(H, a, b)$ dans $\mathscr{E}$ de la façon suivante :

Posons

$$
\mathscr{E}_{1}=\{E \mid E \in \mathscr{E} \text { et }|E| \leqq-b\} \quad \text { et } \quad \mathscr{E}_{2}=\{E \mid E \in \mathscr{E} \text { et }|E|>-b\}
$$

Nous définissons un matroïde $M_{2}(H, a, b)$ dans $\mathscr{E}_{2}$ à l'aide du théorème présenté au paragraphe 2 et de la fonction $f$ définie par

$$
\begin{gathered}
f\left(\mathscr{B}_{2}\right)=a\left\{\left|\bigcup_{B_{2} \in \mathscr{B}_{2}} B_{2}\right|+b\right\} \quad \text { pour } \mathscr{B}_{2} \subset \mathscr{E}_{2} \cdot \mathscr{B}_{2} \neq \varnothing ; \\
f(\varphi)=0 .
\end{gathered}
$$

Cette fonction $f$ satisfait aux conditions du théorème du paragraphe 2, et définit par conséquent un matroïde $M_{2}$ dans $\mathscr{E}_{2}$. 
On étend $M_{2}$ en un matroïde $M(H, a, b)$ dans $\mathscr{E}$ en posant qu'un ensemble $\mathscr{F}(\mathscr{F} \subset \mathscr{E})$ est indépendant dans $M$ si $\mathscr{F}$ satisfait aux deux conditions suivantes :

$1^{\circ} \mathscr{F} \subset \mathscr{E}_{2}$;

$2^{\circ} \mathscr{F}$ est un ensemble indépendant de $M_{2}$.

Remarques : $1^{\circ}$ Le matroïde $M(H, a, b)$ sera indispensable à la résolution d'un problème présenté au paragraphe 6 .

$2^{\circ}$ La construction de $M(H, a, b)$ s'oppose aux constructions " classiques " de matroïdes et doit être rapprochée de la méthode d'Edmonds (" polymatroïdes $")$.

$3^{\circ} M(H, a, b)$ généralise un matroïde particulièrement important et fort connu : dans le cas particulier où $H$ est un hypergraphe 2-uniforme (c'est-à-dire un graphe, noté $G)$, où $a=1$ et où $b=-1$, le matrö̈de $M(G, 1,-1)$ a pour ensembles indépendants les forêts de $G$.

Les cycles élémentaires du graphe $G$ apparaissent comme les ensembles $\mathscr{C}$ $(\mathscr{C} \subset \mathscr{E})$ satisfaisant à

(i) $\left|\bigcup_{C \in \mathscr{C}} C\right|=|\mathscr{C}|$,

et (ii) $\mathscr{C}$ est minimal non vide avec la propriété (i).

\section{INTERSECTION DE MATROÏDES ([3], [4] et [7])}

Soient $k$ matroïdes dans un même ensemble $E$ :

$$
M_{1}\left(E, \mathscr{F}_{1}\right), M_{2}\left(E, \mathscr{F}_{2}\right), M_{3}\left(E, \mathscr{F}_{3}\right), \ldots, M_{k}\left(E, \mathscr{F}_{k}\right) .
$$

L'intersection de ces $k$ matroïdes est par définition l'hypergraphe $H(E, \mathscr{F})$, où $\mathscr{F}=\bigcap_{i=1}^{i=k} \mathscr{F}_{i}$.

L'intersection de deux matroïdes n'est pas nécessairement un matroïde. Par exemple, prenons $E=\left\{e_{1}, e_{2}, e_{3}\right\}$.

$$
\mathscr{F}_{1}=\left\{\varphi,\left\{e_{1}\right\},\left\{e_{2}\right\},\left\{e_{3}\right\},\left\{e_{1}, e_{2}\right\},\left\{e_{2}, e_{3}\right\}\right\}
$$

et

$$
\mathscr{F}_{2}=\left\{\varphi,\left\{e_{1}\right\},\left\{e_{2}\right\},\left\{e_{3}\right\},\left\{e_{1}, e_{2}\right\},\left\{e_{1}, e_{3}\right\}\right\} .
$$

$M_{1}\left(E, \mathscr{F}_{1}\right)$ et $M_{2}\left(E, \mathscr{F}_{2}\right)$ sont deux. matroïdes. Leur intersection $(E, \mathscr{F})$ avec

$$
\mathscr{F}=\left\{\varphi,\left\{e_{1}\right\},\left\{e_{2}\right\},\left\{e_{3}\right\},\left\{e_{1}, e_{2}\right\}\right\}
$$

n'est pas un matroïde.

octobre 1976. 


\section{APPLICATION A DES PROBLÈMES D'AFFECTATION}

\section{a) Une généralisation d'un problème de $D$. Gale [5]}

Soif $H(X, \mathscr{E})$ un hypergraphe où, par exemple, $X$ est un ensemble de travailleurs, $\mathscr{E}$ un ensemble de travaux et où la relation d'incidence sommetsarêtes traduit l'aptitude à effectuer un travail $(x \in E \Leftrightarrow$ le travailleur $x$ est apte à effectuer le travail $E$ ). Tout travailleur peut effectuer un nombre quelconque de travaux, compris entre 0 et $a$. Chaque travail est exécuté par un travailleur ou n'est pas exécuté.

L'employeur doit s'engager à effectuer un ensemble de travaux :

si $b$ est négatif, sous la contrainte qu'il doit se séparer de $(-b)$ travailleurs;

si $b$ est positif, avec la possibilité d'embaucher $b$ travailleurs supplémentaires;

Dans $[5], a=1, b=0$.)

L'ensemble des travaux est totalement ordonné, selon l'intérêt que retire l'entreprise de leur réalisation. Il s'agit, pour l'employeur, de déterminer des ensembles de travaux qu'il pourra mener à bonne fin, et parmi ces ensembles le meilleur.

On peut démontrer (voir [5] ou [8]) que l'ensemble des travaux que l'employeur peut s'engager à mener à bonne fin sous les contraintes précisées coïncide avec l'ensemble des ensembles indépendants du matroïde $M(H, a, b)$.

Par application du théorème du paragraphe 3 , ce problème se ramène donc à la recherche de la meilleure base d'un matroïde.

b) Problème d'affectation à trois dimensions ([4], [5], [6] et [7])

Soit $H(X, \mathscr{E})$ un hypergraphe avec $X=X_{1} \cup X_{2} \cup X_{3}$,

et

$$
\left|X_{i}\right|=k, \quad\left|X_{i} \cap X_{j}\right|=\varnothing, \quad \forall i \neq j,
$$

$$
\left|E \cap X_{i}\right|=1, \quad \forall E \in \mathscr{E} .
$$

Il s'agit de trouver un couplage $G$ maximal (et éventuellement le meilleur si un poids est attribué aux arêtes) : $\operatorname{Max}|G|$, avec

$$
G \subset \mathscr{E} \quad \text { et } \quad \forall E_{k}, \quad E_{j} \in G: \quad E_{k} \cap E_{j}=\varnothing .
$$

Un tel couplage est un ensemble de cardinalité maximale dans l'hypergrapheintersection des trois matroïdes $M_{i}\left(\mathscr{E}, \mathscr{F}_{i}\right)(i=1,2,3)$, où $\mathscr{F}_{i} \in \mathscr{F}_{i}(\mathscr{F} \subset \mathscr{E})$ si et seulement si $\forall E_{k}, E_{j} \in \mathscr{F}: E_{k} \cap E_{j} \cap X_{i}=\varnothing$.

La théorie des matroïdes permet une résolution aisée du problème $a$; son intérêt ėssentiel pour le problème $b$ consiste à mettre en évidence une identité de structure entre ce problème et d'autres problèmes (problème du voyageur de commerce, problèmes de stabilité dans les hypergraphes, ...). 
Nous tenons à remercier le rapporteur pour les remarques extrêmement constructives qu'il a formulées.

\section{BIBLIOGRAPHIE}

1. C. Berge, Graphes et hypergraphes, Dunod, Paris, 1970.

2. H. CRAPO et G. Rota, On the Foundations of Combinatorial Theory : Combinatorial Geometries, M.I.T. Press, Cambridge, 1970.

3. J. Edmonds, Matroids and the Greedy Algorithm, Mathematical Programming, vol. $1, \mathrm{n}^{\circ} 2$, novembre 1971 , p. 127-136.

4. J. Edmonds, Submodular Functions, Matroids, and Certain Polyhedra, Combinatorial Structures and their Applications, Gordon and Breach, Calgary International Conference, juin 1969, p. 69-87.

5. D. Gale, Optimal Assignments in an Ordered Set : an Application of Matroild Theory, Combinatorial Theory, vol. 4, 1968, p. 176-180.

6. P. Hansen et L. Kaufman, A Primal Dual Algorithm for the Three Dimensional Assignment Problem, Cahiers du Centre d'Études de Recherche opérationnelle, vol. 15, n $^{\circ} 3,1973$, p. 327-336.

7. E. LAWLER, Optimal Matroid Intersections, Combinatorial Structures and their Applications, Gordon and Breach, Calgary International Conference, juin 1969, p. 233-234.

8. M. LOREA, Les matroides et leurs applications à des problèmes de recherche opérationnelle, Mémoire, Université de Bruxelles.

9. M. LOREA, Hypergraphes et matroides, Cahiers du Centre d'Études de Recherche opérationnelle, vol. $17, \mathrm{n}^{\circ} 2,1975$.

octobre 1976. 